TRANSACTIONS OF THE

AMERICAN MATHEMATICAL SOCIETY

Volume 352, Number 2, Pages 643-654

$\mathrm{S} 0002-9947(99) 02570-2$

Article electronically published on September 17, 1999

\title{
A HEREDITARILY INDECOMPOSABLE TREE-LIKE CONTINUUM WITHOUT THE FIXED POINT PROPERTY
}

\author{
PIOTR MINC
}

\begin{abstract}
A hereditarily indecomposable tree-like continuum without the fixed point property is constructed. The example answers a question of Knaster and Bellamy.
\end{abstract}

\section{INTRODUCTION}

By a continuum we understand a nondegenerate, connected and compact metric space. A continuum is decomposable if it can be represented as the union of two proper subcontinua. A continuum with no such representation is indecomposable. A continuum containing no decomposable subcontinua is called hereditarily indecomposable. The first example of a hereditarily indecomposable continuum was given by B. Knaster in [15]. A continuum is tree-like if it is the inverse limit of a sequence of trees. This paper is motivated by the question of whether every tree-like hereditarily indecomposable continuum has the fixed point property. The question was asked by Knaster (Problem 69, [6] and Problem 29, [16]) and Bellamy (page $34,[3]$ ). In this paper we answer the question by proving the following theorem. (Note that a fixed point is also a periodic point of period 1.)

Theorem 1.1. For each positive integer $j$ there exists a hereditarily indecomposable tree-like continuum $X_{j}$ and a map $h_{j}: X_{j} \rightarrow X_{j}$ such that $h_{j}$ does not have periodic points of periods less than or equal to $j$.

The question by Knaster and Bellamy is very closely related to the old open problem whether every nonseparating plane continuum has the fixed point property (see [18, Problem 107]). Every nonseparating plane continuum with no interior points is tree-like. In 1978, D. P. Bellamy [2] presented his spectacular example of a (non planar) tree-like continuum without the fixed point property. Indecomposable continua appear naturally in the fixed point problems for both plane and tree-like continua. H. Bell (1967) [1], K. Sieklucki (1968) [28] and S. Iliadis (1970) [14] proved that each fixed-point-free map of a plane nonseparating continuum $X$ into itself must have an invariant indecomposable subcontinuum in the boundary of $X$. The Bellamy continuum is indecomposable. In 1976, R. Mańka [17] proved that every tree-like continuum without the fixed point property must contain an indecomposable continuum, not necessarily invariant (see [23]). Arcs are "the ultimate" decomposable continua. In 1954, K. Borsuk [5] proved the fixed point property for

Received by the editors September 19, 1997.

2000 Mathematics Subject Classification. Primary 54F15; Secondary 54H25.

Key words and phrases. Tree-like continuum, hereditarily indecomposable, fixed points.

This research was supported in part by NSF grant \# DMS-9505108.

(C)1999 American Mathematical Society 
arcwise-connected tree-like continua. The corresponding result in the plane was proven in 1971 by C. Hagopian [9]. Hagopian further extended this line of results by proving the fixed point property for maps preserving arc-components. For the precise statements see [10], [11] (the plane) and [12] (tree-like continua).

Even though the Bellamy continuum is indecomposable, it contains arcs playing an important role in the construction. Very roughly speaking, the idea is to take a map $f$ on some Knaster bucket handle continuum $K$ such that the only fixed point is the endpoint $e$ of $K$. The map $f$ pushes points of the arc-component of $e$ away from $e$. Then, the fixed point $e$ is split into an infinite set $Z$ by replacing an arc containing $e$ by the cone over $Z$. In the fixed-point-free map, branches of the cone are switched while its vertex is pushed further away onto the arc-component of $e$. Bellamy writes in [3]: It seems possible that hereditary indecomposability, like its "opposite," hereditary decomposability, could defeat the split, switch and push strategy. This turns out not to be the case. In this paper we once again (see [20], [22] and [23]) start from Bellamy's marvelous construction to answer his question.

It was observed by J. B. Fugate and L. B. Mohler [8], that if $X$ is a tree-like continuum and $f: X \rightarrow X$ is a fixed-point-free map, then the inverse limit $\tilde{X}$ of copies $X$, with $f$ as the bonding map, is a tree-like continuum and the shift map on $\tilde{X}$ does not have fixed points. The idea of our example is to construct a map $f$ so that the shift homeomorphism on the inverse limit $\widetilde{X}$ is not only fixed-point-free but $\widetilde{X}$ is also hereditarily indecomposable. To get our results as it is stated in Theorem 1.1 , we start our construction from the continuum $B_{j}$ described in [20]. $B_{j}$ is a variation of the Bellamy example admitting a map $f_{j}$ with no periodic points of periods less than or equal to $j$. To get just a fixed-point-free map, one could begin with the original Bellamy's continuum [2] or any of its other variations [25], [26], $[27]$ and [7]. We use the theorem by Fugate and Mohler, to get $\widetilde{B}_{j}$ and $\tilde{f}_{j}$ which is a "smoother" version of $B_{j}$ and $f_{j}$. Every proper subcontinuum of $\widetilde{B}_{j}$ is an arc, a length function can be defined on arcs contained in $\widetilde{B}_{j}$ and $\tilde{f}_{j}$ is a homeomorphism expanding the length of each arc. Then, $\tilde{f}_{j}$ is corrected by a small change in such a way that each arc in $\widetilde{B}_{j}$ produces a hereditarily indecomposable continuum in the inverse limit. For this purpose, we use very extensively the technique presented by W. R. R. Transue and the author in [24] where a transitive map on $[0,1]$ whose inverse limit is the pseudoarc was constructed. The first example of a map on $[0,1]$ whose inverse limit is the pseudoarc was given by G. W. Henderson [13]. However, Henderson's idea could not be used in our construction, because his map is close to the identity and we must keep close to the length expanding map $\tilde{f}_{j}$.

The continuum constructed in this paper is, or at least appears to be, not planar. It would be interesting to prove that every hereditarily indecomposable nonseparating plane continuum has the fixed point property. Such result would parallel the case of weakly chainable continua with a theorem for plane nonseparating continua [19] and a counterexample for tree-like continua [22].

\section{INTRODUCING CROOKEDNESS TO ARCS IN INDECOMPOSABLE CONTINUA}

In this section, we consider a continuum $B$ satisfying the conditions (B1)-(B5) listed below. We use very extensively the technique presented by W. R. R. Transue and the author in [24] to get a map on $B$ whose inverse limit is hereditarily indecomposable. The main goal of this section is Theorem 2.13. It corresponds very 
closely to the theorem on page 1169 in [24] and we have to virtually repeat the same argument to see whether its fine details work in the new setting.

Suppose that $B$ is a tree-like continuum and $\beta$ is a map of $B$ onto $[0,1]$ such that the following conditions are satisfied:

(B1) each (non-trivial) proper subcontinuum of $B$ is an arc,

(B2) $\operatorname{dim}\left(\beta^{-1}(t)\right)=0$ for each $t \in[0,1]$,

(B3) $\beta^{-1}((0,1))$ is homeomorphic to the product $(0,1)$ and the Cantor set,

(B4) $\beta$ restricted to $\beta^{-1}((0,1))$ is the projection of the product onto the $(0,1)$ component, and

(B5) every point of $B$ is either an endpoint or it has a neighborhood homeomorphic to the product of the Cantor set and an open interval. (A point of $B$ is an endpoint if it does not belong to an open arc contained in $B$.)

For an $\operatorname{arc} A \subset B$, we will define the $\beta$-length of $A$ as the sum

$$
\lambda(A)=\sum_{C \in \mathcal{C}(A)} \operatorname{diam}(\beta(C))
$$

where $\mathcal{C}(A)$ denote the set of components of $A \cap \beta^{-1}((0,1))$. Observe that $\lambda(A)=$ $\lambda\left(A_{1}\right)+\lambda\left(A_{2}\right)$ if $A_{1}$ and $A_{2}$ are subarcs of $A$ such that $A=A_{1} \cup A_{2}$ and $A=A_{1} \cap A_{2}$ is a single point.

We will leave the proof of the following proposition to the reader.

Proposition 2.1. For each positive number $\eta$ there is a positive number $\epsilon$ with the property that $\operatorname{diam}(A)<\eta$ for each arc $A \subset B$ such that $\lambda(A)<\epsilon$.

For a point $a \in \beta^{-1}((0,1))$, let $L(a)$ denote the closure of the component of $a$ in $\beta^{-1}((0,1))$. Observe that $\beta$ restricted to $L(a)$ is a homeomorphism onto $[0,1]$. Let $h[a]:[0,1] \rightarrow L(a)$ be the homeomorphism so that $\beta \circ h[a]$ is the identity on $[0,1]$. Let $e(a)$ and $d(a)$ denote the endpoints of $L(a)$ so that $\beta(e(a))=0$ and $\beta(d(a))=1$.

Let $Z=\beta^{-1}\left(\frac{1}{2}\right)$. For each point $z \in Z$ there is at most one point $\tau^{-}(z) \in Z \backslash\{z\}$ such that $e\left(\tau^{-}(z)\right)=e(z)$. In the case when $e(z) \neq e(t)$ for each point $t \in Z \backslash\{z\}$, we will set $\tau^{-}(z)=z$. Similarly, there is at most one point $\tau^{+}(z) \in Z \backslash\{z\}$ such that $d\left(\tau^{+}(z)\right)=d(z)$. In the case when $d(z) \neq d(t)$ for each point $t \in Z \backslash\{z\}$, we will set $\tau^{+}(z)=z$.

Proposition 2.2. Both $\tau^{-}$and $\tau^{+}$are continuous.

Proof. Let $\left(z_{i}\right)_{i=0}^{\infty}$ be a sequence of points of $Z$ converging to some point $z$. Suppose $s$ is the limit of a subsequence of $\left(\tau^{-}\left(z_{i}\right)\right)_{i=0}^{\infty}$. Observe that $e(s)=e(z)$. Thus, either $s=z$ or $s=\tau^{-}(z)$. Suppose $s=z$. In this case, an infinite sequence of sets of the form $L\left(z_{i}\right) \cup L\left(\tau^{-}\left(z_{i}\right)\right)$ converges to $L(z)$. If $\tau^{-}\left(z_{i}\right)=z_{i}, e\left(z_{i}\right)$ is an endpoint. If $\tau^{-}\left(z_{i}\right) \neq z_{i}$, the set $L\left(z_{i}\right) \cup L\left(\tau^{-}\left(z_{i}\right)\right)$ forms a "V" close to $L(z)$. Since there are either infinitely many cases of $\tau^{-}\left(z_{i}\right)=z_{i}$ or infinitely many cases of $\tau^{-}\left(z_{i}\right) \neq z_{i}$, the point $e(z)$ does not have a neighborhood homeomorphic to the product of the Cantor set and an open interval. So, by (B5), $e(z)$ is an endpoint. Thus, $\tau^{-}(z)=z$ and consequently $s=\tau^{-}(z)$. Since the limit of an arbitrary subsequence of $\left(\tau^{-}\left(z_{i}\right)\right)_{i=0}^{\infty}$ is $\tau^{-}(z)$, we have the result that $\lim _{i \rightarrow \infty} \tau^{-}\left(z_{i}\right)=\tau^{-}(z)$. The proof of $\lim _{i \rightarrow \infty} \tau^{+}\left(z_{i}\right)=\tau^{+}(z)$ is similar and will be omitted.

Let $f: B \rightarrow B$ be a map. We will say that $f$ is Lipschitz if there is a constant $s$ such that $\lambda(f(A))<s \lambda(A)$ for each $\operatorname{arc} A \subset B$. If $\sigma$ is a number greater than 1 , we will say that $f$ stretches by $\sigma$ if $\lambda(f(A)) \geq \sigma \lambda(A)$ for each $\operatorname{arc} A \subset B$. 
For any two points $a$ and $b$ belonging to the same arc-component of $B$, let $[a, b]$ denote the $\operatorname{arc}$ in $B$ between $a$ and $b$. ( $[a, a]$ is just a single point.)

Suppose $f_{1}$ and $f_{2}$ are maps of $B$ into itself such that $f_{1}(t)$ and $f_{2}(t)$ belong to the same arc component of $B$ for each $t \in B$. Let $d_{\lambda}\left(f_{1}, f_{2}\right)$ be the supremum of $\lambda\left(\left[f_{1}(t), f_{2}(t)\right]\right)$ where $t \in B$. By saying that $d_{\lambda}\left(f, f^{\prime}\right)$ is finite we will imply, in particular, that $f(t)$ and $f^{\prime}(t)$ belong to the same arc component of $B$ for each $t \in B$.

The following proposition is a simple consequence of Proposition 2.1.

Proposition 2.3. For each positive number $\eta$ there is a positive number $\epsilon$ with the property that the distance between $f$ and $f^{\prime}$ is less than $\eta$ for every two maps $f$ and $f^{\prime}$ of $B$ into itself such that $d_{\lambda}\left(f, f^{\prime}\right)<\epsilon$.

Proposition 2.4. Suppose $f: B \rightarrow B$ is a Lipschitz map with the Lipschitz constant s. Let $F: B \rightarrow B$ be a map such that $d_{\lambda}(f, F)<\eta$ for a certain positive number $\eta$. Then $d_{\lambda}\left(f^{j}, F^{j}\right)<(s+1)^{j-1} \eta$ for each positive integer $j$.

Proof. The proposition is true for $j=1$. We assume that it is true for $j=k$ and we will prove it for $j=k+1$. Let $t$ be an arbitrary point of $B$. Observe that

$$
\lambda\left(\left[f^{k+1}(t), f^{k}(F(t))\right]\right)<s^{k} \lambda([f(t), F(t)])<s^{k} \eta .
$$

Since

$$
\lambda\left(\left[f^{k+1}(t), F^{k+1}(t)\right]\right) \leq \lambda\left(\left[f^{k+1}(t), f^{k}(F(t))\right]\right)+\lambda\left(\left[f^{k}(F(t)), F^{k}(F(t))\right]\right),
$$

we have the result that

$$
\lambda\left(\left[f^{k+1}(t), F^{k+1}(t)\right]\right)<s^{k} \eta+(s+1)^{k-1} \eta<(s+1)^{k} \eta
$$

and the proposition is proven by induction.

For any non-degenerate $\operatorname{arc} A \subset B$, one of its endpoints $a$ and any positive number $\epsilon$, let $H(a, A, \epsilon)$ denote the set of points $t \in B$ such that $a$ and $t$ belong to the same arc component of $B,[a, t] \cap A=\{a\}$ and $\lambda([a, t]) \leq \epsilon$. Clearly, $H(a, A, \epsilon)$ is either the point $a$ or an arc with $a$ as one of its endpoints.

For any arc $A \subset B$, suppose that $a$ and $b$ denote the endpoints of $A$. If $\epsilon$ is a positive number, by $N(A, \epsilon)$ we will denote the union $H(a, A, \epsilon) \cup A \cup H(b, A, \epsilon)$.

Observe that the following proposition holds.

Proposition 2.5. Suppose $f: B \rightarrow B$ is a Lipschitz map with the Lipschitz constant s. Then $f(N(A, \epsilon)) \subset N(f(A), s \epsilon)$ for any arc $A \subset B$ and any positive number $\epsilon$.

For any arc $A \subset B$ and a positive number $\epsilon$ such that $\epsilon<\lambda(A) / 2$, let $K_{1}(A, \epsilon)$ be a subarc of $A$ containing one of the endpoints of $A$ so that $\lambda\left(K_{1}(A, \epsilon)\right)=$ $\lambda(A)-\epsilon$. Let $K_{2}(A, \epsilon)$ be the subarc of $A$ containing the endpoint of $A$ that does not belong to $K_{1}(A, \epsilon)$ so that $\lambda\left(K_{2}(A, \epsilon)\right)=\lambda(A)-\epsilon$. Additionally, let $K(A, \epsilon)=K_{1}(A, \epsilon) \cap K_{2}(A, \epsilon)$.

Let $f: B \rightarrow B$ be a map and let $\epsilon$ and $\delta$ be two positive numbers. We will say that $f$ is $(\epsilon, \delta)$-crooked if for each $\operatorname{arcs} A$ and $C$ contained in $B$ so that $2 \delta<\lambda(A)<\epsilon$ and $f(C)=A$, there are two disjoint arcs $C_{1}$ and $C_{2}$ contained in $C$ so that $f\left(C_{1}\right)=f\left(C_{2}\right)=K(A, \delta)$.

The proof of the next three propositions is left to the reader. 
Proposition 2.6. Let $\epsilon$ and $\delta$ be two positive numbers and let $f: B \rightarrow B$ be a $(\epsilon, \delta)$-crooked map. Then, for each of the arcs $A$ and $C$ contained in $B$ so that $2 \delta<\lambda(A)<\epsilon$ and $f(C)=A$, there are two disjoint arcs $C_{1}^{\prime}$ and $C_{2}^{\prime}$ contained in $C$ so that $f\left(C_{1}^{\prime}\right)=K_{1}(A, \delta)$ and $f\left(C_{1}^{\prime}\right)=K_{2}(A, \delta)$.

Proposition 2.7. If $\alpha \geq \delta$ and $\mu \leq \epsilon$, then every $(\epsilon, \delta)$-crooked map of $B$ into itself is also $(\mu, \alpha)$-crooked.

Proposition 2.8. Let $\epsilon, \delta$ and $\eta$ be positive numbers. Suppose $f$ and $F$ are maps of $B$ into itself such that $d_{\lambda}(f, F)<\eta$. If $f$ is $(\epsilon, \delta)$-crooked, then $F$ is $(\epsilon, \delta+2 \eta)$ crooked.

The following proposition corresponds to [24, Proposition 4].

Proposition 2.9. Let $f$ be a map of $B$ into itself with the property that for each of the positive numbers $\mu$ and $\delta$, there is a positive integer $n$ such that $f^{n}$ is $(\mu, \delta)$ crooked. Then the inverse limit of copies of $B$ with $f$ as the bonding map is hereditarily indecomposable.

Proof. Let $X$ denote the inverse limit of copies of $B$ with $f$ as the bonding map. Let $p_{i}$ denote the projection into the $i$-th copy of $B$ in the inverse sequence. Suppose that $X$ contains two subcontinua $X_{1}$ and $X_{2}$ such that the sets $X_{1} \backslash X_{2}, X_{2} \backslash X_{1}$ and $X_{1} \cap X_{2}$ are not empty. There is a positive integer $k$ such that the sets $p_{k}\left(X_{1}\right) \backslash p_{k}\left(X_{2}\right)$ and $p_{k}\left(X_{2}\right) \backslash p_{k}\left(X_{1}\right)$ are not empty. By $(\mathrm{B} 1), p_{k}\left(X_{1}\right)$ and $p_{k}\left(X_{2}\right)$ are arcs. It follows that $A=p_{k}\left(X_{1}\right) \cup p_{k}\left(X_{2}\right)$ is also an arc. Take $\mu>\lambda(A)$. Let $A_{1}$ denote the closure of $p_{k}\left(X_{1}\right) \backslash p_{k}\left(X_{2}\right)$ and let $A_{2}$ be the closure of $p_{k}\left(X_{2}\right) \backslash p_{k}\left(X_{1}\right)$. Clearly, $A_{1}$ and $A_{2}$ are nondegenerate arcs. Let $\delta$ be a positive number less than the minimum of $\lambda\left(A_{1}\right)$ and $\lambda\left(A_{2}\right)$.

There is a positive integer $n$ such that $f^{n}$ is $(\mu, \delta)$-crooked. Let $C=p_{k+n}\left(X_{1}\right) \cup$ $p_{k+n}\left(X_{2}\right)$. Since $f^{n}(C)=A, C$ is a proper subcontinuum of $B$. Consequently, $C$ is an arc. There are two disjoint arcs $C_{1}$ and $C_{2}$ contained in $C$ such that $f^{n}\left(C_{1}\right)=f^{n}\left(C_{2}\right)=K(A, \delta)$. Since $K(A, \delta)$ is contained in neither $p_{k}\left(X_{1}\right)$ nor $p_{k}\left(X_{2}\right)$, we have the result that, for $i=1,2, C_{i}$ is contained in neither $p_{k+n}\left(X_{1}\right)$ nor $p_{k+n}\left(X_{2}\right)$. It follows that each of the arcs $C_{1}$ and $C_{2}$ contains $p_{k+n}\left(X_{1}\right) \cap p_{k+n}\left(X_{2}\right)$, and thus $C_{1} \cap C_{2} \neq \emptyset$, a contradiction.

The next proposition readily follows from the definitions and Proposition 2.3.

Proposition 2.10. Let $f_{0}, f_{1}, f_{2}, \ldots$ be a sequence of maps of $B$ into itself such that $\sum_{i=0}^{\infty} d_{\lambda}\left(f_{i}, f_{i+1}\right)$ is finite. Then the sequence $f_{0}, f_{1}, f_{2}, \ldots$ converges uniformly and its limit $f$ has the property that $d_{\lambda}\left(f_{0}, f\right) \leq \sum_{i=0}^{\infty} d_{\lambda}\left(f_{i}, f_{i+1}\right)$. Additionally, if each of the maps $f_{0}, f_{1}, f_{2}, \ldots$ is $(\mu, \delta)$-crooked for some positive numbers $\mu$ and $\delta$, then $f$ is also $(\mu, \delta)$-crooked.

The following proposition corresponds to [24, Proposition 5].

Proposition 2.11. Let $\epsilon<1$ and $\gamma<\epsilon / 4$ be two positive numbers. Then there is a Lipschitz map $g: B \rightarrow B$ such that

(i) for each point $t \in B$, $t$ and $g(t)$ are in the same arc component of $B$ and $\lambda([t, g(t)])<\epsilon / 2+\gamma$

(ii) $g$ is $(\epsilon, \gamma)$-crooked,

(iii) $\lambda(g(A)) \geq \lambda(A)$ for each arc $A \subset B$, and if, additionally, $\lambda(A) \geq \gamma$, then 
(iv) $\lambda(g(A))>\epsilon / 2$, and

(v) $g(N(A, r)) \subset N(g(A), r+\gamma)$ for each positive number $r$.

Proof. Let $q$ be an integer such that $q \gamma \geq 4$. Let $\mu=1 / q$ and let $p$ be the integer so that $\mu(p-2)<\epsilon / 2 \leq \mu(p-1)$. We will now define a piecewise linear function $g_{0}:[0,1] \rightarrow[-1,2]$. For each $i=0, \ldots, q-1$, on the interval of the form $[i \mu,(i+1) \mu]$ the function $g_{0}$ is defined in the following way: $g_{0}(i \mu)=i \mu$, then we move up to $(i+p) \mu$ in a crooked fashion, joining linearly values $i \mu,(i+1) \mu$, $(i+2) \mu, \quad(i+1) \mu, \quad(i+2) \mu, \quad(i+3) \mu, \quad(i+2) \mu, \quad(i+1) \mu, \quad(i+2) \mu, \quad(i+3) \mu$, $(i+2) \mu,(i+3) \mu,(i+4) \mu, \ldots,(\operatorname{see}[4])$, then we move crookedly down to $(i-p) \mu$, and then up again to $(i+1) \mu$, a goal which is achieved at $(i+1) \mu$.

Now, we are ready to define $g$. Let $z$ be an arbitrary point of $Z$ and let $t$ be an arbitrary point of $L(z)$. Let

$$
g(t)= \begin{cases}h[z]\left(g_{0}(t)\right), & \text { if } 0 \leq g_{0}(t) \leq 1, \\ h\left[\tau^{+}(z)\right]\left(2-g_{0}(t)\right), & \text { if } g_{0}(t)>1, \\ h\left[\tau^{-}(z)\right]\left(-g_{0}(t)\right), & \text { if } 0>g_{0}(t) .\end{cases}
$$

Continuity of $g$ is guaranteed by Proposition 2.2. It may be verified that $g$ has the remaining required properties.

The following Lemma 2.12 corresponds to the lemma on page 1167 in [24]. The proof presented here is almost the same as in [24]. The only difference, apart from setting it for the continuum $B$ instead of the interval [0,1], is replacing the condition that $f$ eventually expands each interval to $[0,1]$ by the condition that $f$ stretches.

Lemma 2.12. Let $\sigma$ be a number greater than 1 and let $f: B \rightarrow B$ be a Lipschitz map that stretches by $\sigma$. Let $\eta, \delta$ and $\mu$ be positive numbers. Then there is a map $F: B \rightarrow B$ and there is a positive integer $n$ such that

$F$ is Lipschitz and stretches by $\sigma$, $d_{\lambda}(f, F)<\eta$, and $F^{n}$ is $(\mu, \delta)$-crooked.

Proof. Since $f$ is Lipschitz, there is a number $s>2$ such that

$$
\lambda(f(A))<s \lambda(A) \text { for each arc } A \subset B .
$$

Set $\epsilon=\eta / s$. Let $n$ be a positive integer such that $\sigma^{n} \epsilon>2 \mu$. Let $\gamma$ be a positive number less than $\min \left(\epsilon / 4, \delta s^{-n} / 4\right)$.

Let $g$ be a map satisfying Proposition 2.11. Define $F=f \circ g$. Clearly, $F$ is Lipschitz as the composition of two Lipschitz maps. Since $f$ stretches by $\sigma$, it follows from Proposition 2.11 (iii) that $F$ also stretches by $\sigma$.

Suppose $t$ is an arbitrary point of $B$. By Proposition $2.11(\mathrm{i}), t$ and $g(t)$ are in the same arc component of $B$ and $\lambda([g(t), t])<\epsilon=\eta / s$. It follows that $F(t)$ and $f(t)$ are in the same arc component of $B$ and, by $(1), \lambda([F(t), f(t)])<\eta$.

To prove that $F^{n}$ is $(\mu, \delta)$-crooked, we need the following two claims.

Claim 1. Let $A \subset B$ be an arc such that $\lambda(A) \geq \gamma$. Let $r$ be a positive real number and let $j$ be a positive integer. Then $F^{j}(N(A, r)) \subset N\left(F^{j}(A), q\right)$, where $q=s^{j}(r+2 \gamma)$.

Proof of Claim 1. Since $F$ stretches by $\sigma>1$, we have the result that $\lambda\left(F^{i}(A)\right)>$ $\gamma$ for each positive integer $i$. Set $q_{0}=r$ and $q_{i+1}=s\left(q_{i}+\gamma\right)$ for $i=0,1, \ldots$ Repeatedly using Proposition $2.11(\mathrm{v})$, the condition (1) and Proposition 2.5, one 
can prove $F^{j}(N(A, r)) \subset N\left(F^{j}(A), q_{j}\right)$. Observe that $q_{j}=s^{j} r+s^{j} \gamma+s^{j-1} \gamma+$ $\cdots+s \gamma=s^{j} r+\gamma s \frac{s^{j}-1}{s-1}=s^{j}\left(r+\gamma \frac{s}{s-1} \frac{s^{j}-1}{s^{j}}\right)<s^{j}\left(r+\gamma \frac{s}{s-1}\right)$. Since $s>2$, $\frac{s}{s-1}<2$, and, consequently, $q_{j}<s^{j}(r+2 \gamma)$, so the claim is true.

Claim 2. Let $C \subset B$ be an arc such that $2 \gamma<\lambda(C)<\epsilon+2 \nu$ for some positive number $\nu$. Then there is an arc $G \subset C$ such that $2 \gamma<\lambda(G)<\epsilon$ and $C \subset N(G, \nu)$.

Proof of Claim 2. If $\lambda(C)<\epsilon$, we set $G=C$. So, we may assume that $\lambda(C) \geq \epsilon$. Since $\lambda(C)-\epsilon<2 \nu$, there is a number $\kappa>\lambda(C)-\epsilon$ such that $\kappa<\lambda(C)-\epsilon+\gamma$ and $\kappa<2 \nu$. Set $G=K(C, \kappa / 2)$. In this case, $\lambda(G)=\lambda(C)-\kappa<\epsilon$. We have also $\lambda(G)=\lambda(C)-\kappa>\epsilon-\gamma>2 \gamma$. Finally, $C=N(G, \kappa / 2) \subset N(G, \nu)$.

Let $A$ be a subarc of $B$ such that $2 \delta<\lambda(A)<\mu$. Suppose that $I \subset B$ is an arc such that $F^{n}(I)=A$. To complete the proof of the lemma, it is enough to show that

there are two disjoint subarcs $A_{1}$ and $A_{2}$ of $I$ such that

$$
K(A, \delta) \subset F^{n}\left(A_{1}\right) \cap F^{n}\left(A_{2}\right) .
$$

We will observe that

$$
\lambda(I)<\gamma
$$

Suppose the contrary. Then, by Proposition 2.11 (iv), $\lambda(g(I))>\epsilon / 2$. Since $f$ stretches by $\sigma, \lambda(F(I))>\sigma \epsilon / 2$. Since $F$ stretches by $\sigma, \lambda\left(F^{n}(I)\right)>\sigma^{n} \epsilon / 2$. The last number is greater than $\mu$ by the choice of $n$, so we get $\lambda(A)>\mu$, a contradiction.

Since $F^{n}(I)=A$ and $\lambda(A)>2 \delta>\gamma, \lambda\left(F^{n}(I)\right)>\gamma$. Let $m$ be the greatest integer such that $\lambda\left(F^{m}(I)\right)<\gamma$. Clearly, $0 \leq m<n$. Denote $F^{m}(I)$ by $M$. We will consider two cases: $\lambda(g(M))>2 \gamma$ and $\lambda(g(M)) \leq 2 \gamma$.

Case. $\lambda(g(M))>2 \gamma$.

Since $\lambda(M)<\gamma$, it follows from Proposition 2.11 (i) that $\lambda(g(M))<\epsilon+3 \gamma$. It follows from Claim 2 that there is an arc $G \subset g(M)$ such that $2 \gamma<\lambda(G)<\epsilon$ and $g(M) \subset N(G, 2 \gamma)$. Since $g$ is $(\epsilon, \gamma)$-crooked (Proposition 2.11 (ii)), it follows from Proposition 2.6 that there are two disjoint arcs $M_{1}$ and $M_{2}$ contained in $M$ such that $g\left(M_{1}\right)=K_{1}(G, \gamma)$ and $g\left(M_{2}\right)=K_{2}(G, \gamma)$. There are two disjoint arcs $A_{1}$ and $A_{2}$ contained in $I$ such that $F^{m}\left(A_{k}\right)=M_{k}$ for $k=1,2$. Observe that $g(M) \subset N\left(g\left(M_{k}\right), 3 \gamma\right)$ and it follows from Proposition 2.5 that $F(M) \subset N\left(F\left(M_{k}\right), 3 s \gamma\right)$ for $k=1,2$. Since $\lambda\left(g\left(M_{k}\right)\right)>\gamma$ and $f$ stretches (by $\left.\sigma\right)$, we have the result that $\lambda\left(F\left(M_{k}\right)\right)>\gamma$. It follows from Claim 1 that $A=F^{n}(I)=$ $F^{n-m}(M) \subset N\left(F^{n-m}\left(M_{k}\right), s^{n-m-1}(3 s \gamma+2 \gamma)\right)$. Since $s^{n-m-1}(3 s \gamma+2 \gamma)<$ $4 s^{n} \gamma<\delta, K(A, \delta) \subset F^{n-m}\left(M_{1}\right) \cap F^{n-m}\left(M_{2}\right)=F^{n}\left(A_{1}\right) \cap F^{n}\left(A_{2}\right)$.

Case. $\lambda(g(M)) \leq 2 \gamma$.

It follows from (1) and the choice of $m$ that $\gamma \leq \lambda(F(M))<2 \gamma s$. (Notice, that the last inequality implies, in particular, that $n>m+1$, because $\gamma s<\delta$.)

By Proposition 2.11 (i) and (iv), $\epsilon / 2<\lambda(g(F(M)))<\epsilon+2 \gamma(s+1)$. Using Claim 2 we get an $\operatorname{arc} G \subset g(F(M))$ such that $2 \gamma<\lambda(G)<\epsilon$ and $g(F(M)) \subset$ $N(G, \gamma(s+1))$. Since $g$ is $(\epsilon, \gamma)$-crooked (Proposition 2.11 (ii)), it follows from Proposition 2.6 that there two disjoint arcs $M_{1}$ and $M_{2}$ contained in $F(M)$ such that $g\left(M_{1}\right)=K_{1}(G, \gamma)$ and $g\left(M_{2}\right)=K_{2}(G, \gamma)$. There are two disjoint arcs 
$A_{1}$ and $A_{2}$ contained in $I$ such that $F^{m+1}\left(A_{k}\right)=M_{k}$ for $k=1,2$. Observe that $g(F(M)) \subset N\left(g\left(M_{k}\right), \gamma(s+1)+\gamma\right) \subset N\left(g\left(M_{k}\right), 2 s \gamma\right)$ and it follows from Proposition 2.5 that $F^{2}(M) \subset N\left(F\left(M_{k}\right), 2 s^{2} \gamma\right)$ for $k=1,2$. Since $\lambda\left(g\left(M_{k}\right)\right)>\gamma$ and $f$ stretches (by $\sigma$ ), we have the result that $\lambda\left(F\left(M_{k}\right)\right)>\gamma$. It follows from Claim 1 that $A=F^{n}(I)=F^{n-m}(M) \subset N\left(F^{n-m-1}\left(M_{k}\right), s^{n-m-2}\left(2 s^{2} \gamma+2 \gamma\right)\right)$. Since $s^{n-m-2}\left(2 s^{2} \gamma+2 \gamma\right)<4 s^{n} \gamma<\delta, K(A, \delta) \subset F^{n-m}\left(M_{1}\right) \cap F^{n-m}\left(M_{2}\right)=$ $F^{n}\left(A_{1}\right) \cap F^{n}\left(A_{2}\right)$ and the proof of the lemma is complete.

Theorem 2.13. Suppose $\epsilon$ is a positive number and $f_{0}: B \rightarrow B$ is a Lipschitz map that stretches by a certain number $\sigma>1$. Then there is a map $f: B \rightarrow B$ such that $d_{\lambda}\left(f, f_{0}\right)<\epsilon$ and the inverse limit of copies of $B$ with $f$ as the bonding map is hereditarily indecomposable.

Proof. We are going to construct a sequence of positive integers $n(1), n(2), n(3)$, $\ldots$ and a sequence $f_{1}, f_{2}, f_{3}, \ldots$ of maps of $B$ into itself such that, for each positive integer $i$, the following conditions are satisfied:

(i) $f_{i}$ is a Lipschitz map stretching by $\sigma$,

(ii) $d_{\lambda}\left(f_{i-1}, f_{i}\right)<2^{-i} \epsilon$, and

(iii) $f_{i}^{n(k)}$ is $\left(k, 2^{-k}-2^{-k-i}\right)$-crooked for each positive integer $k \leq i$.

To construct $f_{1}$, we apply Lemma 2.12 with $f=f_{0}, \eta=\epsilon / 2, \delta=2^{-2}$ and $\mu=1$. We set $f_{1}=F$ and $n(1)=n$. We assume that $n(1), n(2), \ldots, n(i-1)$ and $f_{1}, f_{2}, \ldots, f_{i-1}$ have already been constructed, and we will construct $n(i)$ and $f_{i}$.

By Proposition 2.4, there is a positive number $\eta<2^{-i} \epsilon$ such that, if $F: B \rightarrow B$ is a map with the property $d_{\lambda}\left(f_{i-1}, F\right)<\eta$, then $d_{\lambda}\left(f_{i-1}^{n(k)}, F^{n(k)}\right)<2^{-k-i-1}$ for each positive integer $k<i$. Now, use Lemma 2.12 with $f=f_{i-1}, \delta=2^{-i}-2^{-i-i}$ and $\mu=i$. Define $f_{i}=F$ and $n(i)=n$. Clearly, the conditions (i), (ii) and (iii) for $k=i$ are satisfied. By Proposition 2.8, the choice of $\eta$ guarantees that $f_{i}^{n(k)}=F^{n(k)}$ is $\left(k, 2^{-k}-2^{-k-i}\right)$-crooked for $k<i$. So the construction is complete.

By (ii) and Proposition 2.10, the sequence $f_{0}, f_{1}, f_{2}, \ldots$ converges uniformly. Denote the limit by $f$. By Proposition $2.10, d_{\lambda}\left(f, f_{0}\right)<\epsilon$. By (iii) and Proposition 2.7, $f_{i}^{n(k)}$ is $\left(k, 2^{-k}\right)$-crooked for every one of the positive integers $k$ and $i$ such that $k \leq i$. By the second part of Proposition 2.10, $f^{n(k)}$ is $\left(k, 2^{-k}\right)$-crooked for every positive integer $k$. Applying Propositions 2.7 and 2.9, we get the result that the inverse limit of copies of $B$ with $f$ as the bonding map is hereditarily indecomposable.

\section{THE MAIN RESULT}

In this section we finish our proof of Theorem 1.1 by supplying a suitable continuum $B$ to the machinery developed in the previous section. For this purpose we will take $\widetilde{B}_{j}$ as it is described in [21]. The continuum $\widetilde{B}_{j}$ results from applying the theorem by Fugate and Mohler [8] to the continuum $B_{j}$ described in [20]. As we noted in the introduction, to get just a fixed-point-free map in Theorem 1.1, one could replace $B_{j}$ with the original Bellamy's continuum [2] or any of its other variations [25], [26], [27] and [7]. Even though the properties required in Section 2 are quite apparent for any of these continua, we feel obliged to check the details at least in the case of $B_{j}$. To do that we need to summarize the construction from [20] and Section 2 of [21]. 
For each positive integer $k$, let $g_{k}:[0,1] \rightarrow[0,1]$ be the function stretching the interval $[0,1] k$ times and then folding it uniformly back onto itself. For example, $g_{2}$ is the roof-top map on $[0,1]$. For each positive integer $n$, let $S_{n}$ be the inverse limit of the inverse system of copies of $[0,1]$ with every bonding map equal to $g_{n}$. Let $p_{n}^{k}$ be the projection of $S_{n}$ onto the $k$-th element of the inverse system. Let $e_{n}$ denote the point $(0,0, \ldots)$ and let $d_{n}$ denote the point $\left(1,1 / n, 1 / n^{2}, 1 / n^{3}, \ldots\right)$. Let $J_{n}$ denote the arc in $S_{n}$ between $e_{n}$ and $d_{n}$. Let $g$ denote the map from $S_{n}$ onto itself induced by $g_{2}$, i.e. $g\left(\left(x_{0}, x_{1}, \ldots\right)\right)=\left(g_{2}\left(x_{0}\right), g_{2}\left(x_{1}\right), \ldots\right)$.

The following properties of $S_{n}$ are very well known.

(S1) Every proper subcontinuum of $S_{n}$ is an arc.

(S2) For each arc $A \subset S_{n}$, there is a positive integer $m_{0}$ such that $g^{m}\left(d_{n}\right) \notin A$ for each $m \geq m_{0}$.

If $n$ is even, we additionally have:

(S3) $e_{n}$ is the only endpoint of $S_{n}$,

(S4) each point of $S_{n} \backslash\left\{e_{n}\right\}$ has a neighborhood homeomorphic to the product of the Cantor set and an open interval,

(S5) $g$ is a homeomorphism, and

(S6) $g^{-1}\left(J_{n}\right) \subset J_{n} \backslash\left\{d_{n}\right\}$.

For each positive integer $j, n(j)$ denotes $2\left(4^{1}-1\right)\left(4^{2}-1\right) \ldots\left(4^{j}-1\right)$. Ву a slight variation of the original Bellamy's construction [2], it was proven in [20] that there is a tree-like continuum $B_{j}$ and there is a continuous map $f_{j}: B_{j} \rightarrow B_{j}$ without periodic points of periods less than or equal to $j$. Roughly speaking, $B_{j}$ was obtained by replacing $J_{n(j)}$ in $S_{n(j)}$ by a cone over some zero dimensional set $Z_{j}$. More precisely, there is a continuous map $q_{j}$ (this map was denoted by $q$ in [20]) of $B_{j}$ onto $S_{n(j)}$ with the following properties:

(Be1) $q_{j}^{-1}(x)$ is an one-point set for each $x \in\left(S_{n(j)} \backslash J_{n(j)}\right) \cup\left\{d_{n(j)}\right\}$.

(Be2) The set $Z_{j}=q_{j}{ }^{-1}\left(e_{n(j)}\right)$ is zero-dimensional.

(Be3) $q_{j}^{-1}\left(J_{n(j)}\right)$ is a cone over $Z_{j} \cdot q_{j}^{-1}\left(J_{n(j)}\right)$ is nowhere dense in $B_{j}$. If $\tilde{d}$ denotes the vertex of the cone and, for each $z \in Z_{j}, A_{z}$ denotes the arc between $z$ and $\tilde{d}$, then $q_{j}$ restricted to $A_{z}$ is a homeomorphism onto $J_{n(j)}$.

(Be4) $q_{j} \circ f_{j}=g \circ q_{j}$.

It follows that

(Be5) every proper subcontinuum of $B_{j}$ is arcwise connected, and

(Be6) if $C$ is a subcontinuum of $B_{j}$ such that $\tilde{d} \notin C$, then $C$ is an arc and $f_{j}$ restricted to $C$ is a homeomorphism.

The continuum $B_{j}$ cannot be used as $B$ is Section 2 . For instance, not every proper subcontinuum of $B_{j}$ is an arc. To get a continuum that is more suitable for our purpose, we use the technique presented by J. B. Fugate and L. B. Mohler in [8]. As in [21], let $\widetilde{B}_{j}$ be the inverse limit of the inverse system of copies of $B_{j}$ with the bonding maps equal to $f_{j}$. Let $\tilde{p}_{j}^{k}$ be the projection of $\widetilde{B}_{j}$ onto the $k$-th element of the inverse system. Let $\tilde{f}_{j}$ denote the right shift on $\widetilde{B}_{j}$, i.e. $\tilde{f}_{j}\left(\left(b_{0}, b_{1}, b_{2}, \ldots\right)\right)=$ $\left(f_{j}\left(b_{0}\right), b_{0}, b_{1}, b_{2}, \ldots\right)$.

Let $\pi_{j}$ denote the map $q_{j} \circ \tilde{p}_{j}^{0}$. Observe that the following proposition is true.

Proposition 3.1. $\pi_{j}{ }^{-1}(x)$ is zero-dimensional for each $x \in S_{n(j)}$. 
Proposition 3.2. Let $A_{0}$ be an arc contained in $S_{n(j)}$ and let $C$ be a component of $\pi_{j}{ }^{-1}\left(A_{0}\right)$. Then, $\pi_{j}$ restricted to $C$ is a homeomorphism onto $A_{0}$.

Proof. For each $k=1,2, \ldots$, let $A_{k}=g^{-k}\left(A_{0}\right)$. Since $g$ is a homeomorphism $A_{k}$ is an arc. By (S2), there is an integer $m$ such that $d_{n(j)} \notin A_{k}$ for each integer $k \geq m$.

By $(\mathrm{Be} 4), g^{k} \circ q_{j} \circ \tilde{p}_{j}^{k}=\pi_{j}$ and consequently $q_{j} \circ \tilde{p}_{j}^{k}(C) \subset A_{k}$. Let $C_{k}$ be the component of $q_{j}^{-1}\left(A_{k}\right)$ containing $\tilde{p}_{j}^{k}(C)$. Observe that $C$ is the inverse limit of $C_{k}$ 's.

Clearly, $\tilde{d} \notin C_{k}$ for each integer $k \geq m$. By (Be6), $C_{k}$ is an arc and the bonding map $f_{j}$ restricted to $C_{k}$ is a homeomorphism for each integer $k \geq m$. It follows that $C$ is also an arc and $\tilde{p}_{j}^{m}$ restricted to $C$ is a homeomorphism onto $C_{m}$. By (Be1) and (Be3), $q_{j}$ restricted to $C_{m}$ is a homeomorphism onto $A_{m}$. Since $g^{m}$ restricted to $A_{m}$ is a homeomorphism onto $A_{0}$, we have the result that $\pi_{j}=g^{m} \circ q_{j} \circ \tilde{p}_{j}^{m}$ restricted to $C$ is a homeomorphism onto $A_{0}$.

Proposition 3.3. Every proper subcontinuum of $\widetilde{B}_{j}$ is an arc.

Proof. Let $C$ be a proper subcontinuum of $\widetilde{B}_{j}$. Let $C_{k}=\tilde{p}_{j}^{k}(C)$ for each integer $k=0,1, \ldots$. Since $C_{k}$ must be a proper subcontinuum of $B_{j}$ for sufficiently large $k$, it follows from (Be5) that $C_{k}$ is be a proper subcontinuum of $B_{j}$ for each $k$. By $(\mathrm{Be} 5)$ and (S1), $A=q_{j}\left(C_{0}\right)$ is an arc. Since $C \subset \pi_{j}^{-1}\left(C_{0}\right)$, it follows from (3.2) that $C$ is also an arc.

Proposition 3.4. Let $s$ be a point of the interval $[0,1]$. Suppose $\kappa$ is a map of a compactum $M$ onto $[0,1]$ such that

1. $\kappa^{-1}(s)$ is zero-dimensional, and

2. $\kappa$ restricted to each component of $M$ is a homeomorphism onto $[0,1]$.

Then, $M$ is homeomorphic to the product of $[0,1]$ and $\kappa^{-1}(s)$.

Proof. For any $x \in M$, let $\sigma(x)$ denote the only point from the component of $x$ in $M$ such that $\kappa(\sigma(x))=s$. Let $\varphi: M \rightarrow[0,1] \times \kappa^{-1}(s)$ be defined by $\varphi(x)=$ $(\kappa(x), \sigma(x))$. We will prove that $\varphi$ is a homeomorphism of $M$ onto $[0,1] \times \kappa^{-1}(s)$.

Let $x_{1}, x_{2}, \ldots$ be a sequence of points of $M$ converging to some point $x \in M$. We will prove that

$$
\lim _{i \rightarrow \infty} \sigma\left(x_{i}\right)=\sigma(x) .
$$

Since $M$ is compact, there is an infinite subsequence $\sigma\left(x_{i(1)}\right), \sigma\left(x_{i(2)}\right), \ldots$ converging to some point $y \in \kappa^{-1}(s)$. Since $M$ is compact, $\lim _{k \rightarrow \infty} x_{i(k)}=x$ and $\lim _{k \rightarrow \infty} \sigma\left(x_{i(k)}\right)=y, x$ and $y$ must belong to the same component of $M$. Since $\sigma(x)$ is the only point of the component of $x$ in $M$ belonging to $\kappa^{-1}(s)$, we have the result that $y=\sigma(x)$. Thus, $\sigma(x)$ is the limit of any convergent subsequence of $\sigma\left(x_{1}\right), \sigma\left(x_{2}\right), \ldots,(*)$ is true and consequently $\sigma: M \rightarrow \kappa^{-1}(s)$ is continuous. It follows that $\varphi$ is also continuous. As an one-to-one continuous function defined on a compactum, $\varphi$ is a homeomorphism.

Let $\beta_{j}: \widetilde{B}_{j} \rightarrow[0,1]$ be the map $p_{n(j)}^{0} \circ q_{j} \circ \tilde{p}_{j}^{0}$.

Proposition 3.5. The conditions (B1)-(B5) from Section 2 are satisfied by $B=$ $\widetilde{B}_{j}$ and $\beta=\beta_{j}$. Moreover, if $\lambda$ is the $\beta_{j}$-length on $\widetilde{B}_{j}$, then $\lambda\left(\tilde{f}_{j}(A)\right)=2 \lambda(A)$ for each arc $A \subset \widetilde{B}_{j}$. 
Proof. (B1) follows from Proposition 3.3. (B2) follows from Proposition 3.1. (B3) and (B4) are proven in Proposition 2.11, [21].

To prove that (B5) is also satisfied, suppose $x \in \widetilde{B}_{j}$ is not an endpoint. By Proposition $3.2, \pi_{j}(x) \neq e_{n(j)}$. By (S4), there is a set $T \subset S_{n(j)}$ containing $\pi_{j}(x)$ in its interior and homeomorphic to the product of $[0,1]$ and the Cantor set. By Propositions 3.4 and $3.2, \pi_{j}^{-1}(T)$ is homeomorphic to the product of $[0,1]$ and the Cantor set. Since $x$ belongs to the interior of $\pi_{j}^{-1}(T), x$ has a neighborhood homeomorphic to the product of the open interval $(0,1)$ and the Cantor set.

The remainder of the proposition follows readily from Proposition 2.12, [21].

Proof of Theorem 1.1. Let $\lambda$ be the $\beta_{j}$-length on $\widetilde{B}_{j}$.

By Proposition 2.9, [21], the map $\tilde{f}_{j}$ does not have periodic points of periods less than or equal to $j$. There is a positive number $\eta$ such that any map $f: \widetilde{B}_{j} \rightarrow \widetilde{B}_{j}$ that is $\eta$ close to $\tilde{f}_{j}$ does have periodic points of periods less than or equal to $j$. Chose $\epsilon>0$ so that the distance between $f$ and $\tilde{f}_{j}$ is less than $\eta$ for every map $f: \widetilde{B}_{j} \rightarrow \widetilde{B}_{j}$ such that $d_{\lambda}\left(f, \tilde{f}_{j}\right)<\epsilon$ (see Proposition 2.3).

By Proposition 3.5, we may use Theorem 2.13 with $B=\widetilde{B}_{j}, \beta=\beta_{j}$ and $f_{0}=\tilde{f}_{j}$. Let $f: \widetilde{B}_{j} \rightarrow \widetilde{B}_{j}$ be the map resulting from Theorem 2.13. Define $X_{j}$ as the inverse limit of copies of $\widetilde{B}_{j}$ with $f$ as the bonding map and let $h_{j}: X_{j} \rightarrow X_{j}$ be the right shift map. Observe that $X_{j}$ is a tree-like continuum as the inverse limit of tree-like continua. By Theorem 2.13, $X_{j}$ is hereditarily indecomposable. By the choice of $\epsilon$, $f$ does not have periodic points of periods less than or equal to $j$. Since periodic points of $h_{j}$ correspond to those of $f$, we infer that $h_{j}$ does not have periodic points of periods less than or equal to $j$.

\section{REFERENCES}

1. H. Bell, On fixed point properties of plane continua, Trans. Amer. Math. Soc. 128 (1967), 539-548. MR 35:4888

2. D.P. Bellamy, A tree-like continuum without the fixed-point property, Houston J. Math. 6 (1980), 1-13. MR 81h:54039

3. book (H. Cook et al., eds.), Marcel Dekker, New York, 1995, pp. 27-35. MR 96a:54056

4. R. H. Bing, A homogeneous indecomposable plane continuum, Duke Math. J. 15 (1948), 729-742. MR 10:261a

5. K. Borsuk, A theorem on fixed points, Bull. Acad. Sci. Polon. 2 (1954), 17-20. MR 16:275h

6. H. Cook, W. T. Ingram and A. Lelek, A list of problems known as Houston Problem Book, in Continua with the Houston problem book (H. Cook et al., eds.), Marcel Dekker, New York, 1995, pp. 365-398. MR 96f:54042

7. L. Fearnley and D. G. Wright, Geometric realization of a Bellamy continuum, Bull. London Math. Soc. 25 (1993), 177-183. MR 94b:54095

8. J. B. Fugate and L. A. Mohler, A note on fixed points in tree-like continua, Topology Proc. 2 (1977), 457-460. MR 80k:54062

9. C. L. Hagopian, A fixed point theorem for plane continua, Bull. Amer. Math. Soc. 77 (1971), 351-354. MR 42:8469

10. , Fixed points of plane continua, Rocky Mountain J. Math. 23 (1993), 119-186. MR 94h:54054

11. Soc. 348 (1996), 4525-4548. MR 97a:54047

12. $\quad$ The fixed point property for deformations of tree-like continua, Fund. Math. 155 (1998), 161-176. MR 99b:54046

13. G. W. Henderson, The pseudo-arc as an inverse limit with one binding map, Duke Math. J. 31 (1964), 421-425. MR 29:4039 
14. S. Iliadis, Positions of continua in a plane and fixed points, Vestn. Moskov. Univ. 25 (1970), 66-70. MR 44:4726

15. B. Knaster, Un continu dont tout sous-continu est indécomposable, Fund. Math. 3 (1922), $247-286$.

16. W. Lewis, Continuum theory problems, Topology Proc. 8 (1983), 361-394. MR 86a:54038

17. R. Mańka, Association and fixed points, Fund. Math. 91 (1976), 105-121. MR 54:1183

18. R. D. Mauldin (ed.), The Scottish Book: Mathematics from the Scottish Café, Birkhauser, Boston, 1981. MR 84m:00015

19. P. Minc, A fixed point theorem for weakly chainable plane continua, Trans. Amer. Math. Soc. 317 (1990), 303-312. MR 90d:54067

20. $ـ$ A tree-like continuum admitting fixed point free maps with arbitrarily small trajectories, Topology and its Appl. 46 (1992), 99-106. MR 94a:54108

21. _ A periodic points free homeomorphism of a tree-like continuum, Trans. Amer. Math. Soc. 348 (1996), 1487-1519. MR 96h:54029

22. $ـ$ A weakly chainable tree-like continuum without the fixed point property, Trans. Amer. Math. Soc. 351 (1999), 1109-1121. MR 99e:54024

23. _ A self map of a tree-like continuum with no invariant indecomposable subcontinuum, preprint.

24. P. Minc and W. R. R. Transue, A transitive map on $[0,1]$ whose inverse limit is the pseudoarc, Proc. Amer. Math. Soc. 111 (1991), 1165-1170. MR 91g:54050

25. L.G. Oversteegen and J.T. Rogers, Jr., Tree-like continua as limits of cyclic graphs, Topology Proc. 4 (1979), 507-515. MR 82c:54032

26. __ An inverse limit description of an atriodic tree-like continuum and an induced map without a fixed point, Houston J. Math. 6 (1980), 549-564. MR 82j:54075

27. _ Fixed-point-free maps on tree-like continua, Topology and its Appl. 13 (1982), 85-95. MR 83b:54044

28. K. Sieklucki, On a class of plane acyclic continua with the fixed point property, Fund. Math. 63 (1968), 257-278. MR 39:2139

Department of Mathematics, Auburn University, Auburn, Alabama 36849

E-mail address: mincpio@mail.auburn.edu 